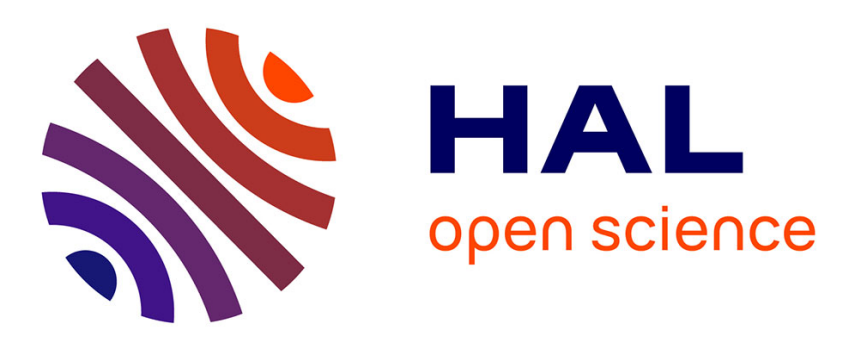

\title{
Evaporation of an emulsion trapped in a yield stress fluid
}

Geoffroy Guéna, Joëlle Corde, Sarah Fouilloux, Jean-Baptise d'Espinose, François Lequeux, Laurence Talini

\section{To cite this version:}

Geoffroy Guéna, Joëlle Corde, Sarah Fouilloux, Jean-Baptise d'Espinose, François Lequeux, et al.. Evaporation of an emulsion trapped in a yield stress fluid. European Physical Journal E: Soft matter and biological physics, 2009, 28, pp.463-468. hal-00547825

\section{HAL Id: hal-00547825 \\ https://hal.science/hal-00547825}

Submitted on 17 Dec 2010

HAL is a multi-disciplinary open access archive for the deposit and dissemination of scientific research documents, whether they are published or not. The documents may come from teaching and research institutions in France or abroad, or from public or private research centers.
L'archive ouverte pluridisciplinaire HAL, est destinée au dépôt et à la diffusion de documents scientifiques de niveau recherche, publiés ou non, émanant des établissements d'enseignement et de recherche français ou étrangers, des laboratoires publics ou privés. 


\title{
Evaporation of an emulsion trapped in a yield stress fluid
}

\author{
G. Guéna ${ }^{\mathrm{a}}$, J. Corde, S. Fouilloux, J.-B. d'Espinose, F. Lequeux, and L. Talini
}

PPMD-ESPCI, UMR 7615, 10 rue Vauquelin, 75231 Paris Cedex 05, France

\begin{abstract}
The present work deals with emulsions of volatile alkanes in an aqueous clay suspension, Laponite, which forms a yield stress fluid. For a large enough yield stress (i.e. Laponite concentration), the oil droplets are prevented from creaming and the emulsions are thus mechanically stabilized. We have studied the evaporation kinetics of the oil phase of those emulsions in contact with the atmosphere. We show that the evaporation process is charscterized by the formation of a sharp front separating the emulsion from a droplet-free Laponite phase, and that the displacement of the front vs. time follows a diffusion law. Experimental data are confronted to a diffusion-controlled model, in the case where the limiting step is the diffusion of the dissolved oil through the aqueous phase. The nature of the alkane, as well as its volume fraction in the emulsion, has been varied. Quantitative agreement with the model is achieved without any adjustable parameter and we describe the mechanism leading to the formation of a front.
\end{abstract}

\section{Introduction}

Emulsions provide an efficient way to transport and/or store small quantities of a chemical compound in a large volume of an immiscible liquid. Due to a high surface-tovolume ratio, and thus a high energetical cost associated with their formation, these media are not a stable state of matter. Therefore, kinetic stabilization of emulsions is crucial and efficient stabilization processes are sought for. In the case of surfactant-free emulsions, demixing of the two phases occurs over short times (typically less than 24 hours) which leads to the development of a supernatant. When emulsions are stabilized with surfactants, diffusion-controlled destabilization such as Ostwald ripening can be observed over long time scales [1]. Recently, it has been emphasized that the volatility of either the emulsifying or the emulsified phase can govern the long time evolution of an emulsion $[2,3]$.

In this paper, we address the specific question of the evaporation of the oil contained in an oil/water emulsion whose continuous phase is a colloidal clay suspension that forms an aging yield stress fluid. The fluid aging induces a growing yield stress with the time elapsed after emulsification. Here, the time scale of the yield stress increase is short compared to the destabilization processes of the emulsion: coalescence, creaming and diffusion-controlled relaxation. The advantage of such emulsions is twofold. First: as the droplets are prevented from creaming dur-

\footnotetext{
a-mail: geoffroy-guena@espci.fr
}

ing the whole duration of the experiment, the effects due to evaporation are not hindered or modified by hydrodynamic ones. Second, there is no need for the addition of any surfactant in the system ${ }^{1}$. The originality of our experiment is that during the evaporation process, we observe (and explain why we can observe) the formation of a front separating the emulsion from a droplet-free aqueous phase. As a consequence, we show that the kinetics of evaporation is controlled in an unconventional manner by the concentration profile of alkane.

\section{Experimental set-up}

Emulsifying phase: The emulsifying phase is an aqueous suspension of Laponite RD (Rockwood) of concentration $\phi_{m}=2.5 \% \mathrm{w} / \mathrm{w}$. The $p \mathrm{H}$ is set to 10 by adding hydroxide sodium in order to avoid chemical dissolution of the clay particles $[4,5]$. The dispersion is stirred for 72 hours to ensure full dispersion and exfoliation of the clay platelets. It is further let at rest for 24 hours, the fluid then evolves from a low-viscosity liquid to a paste with a large apparent yield stress within a few hours. It can then be "rejuvenated", i.e. recover its liquid state by application of a strong shear [6].

Emulsified phase: The emulsified phase is a light linear alkane whose vapor pressure is comparable to but smaller

\footnotetext{
1 It is unclear whether the presence of a third compound may affect or not the evaporation process [1].
} 
Table 1. Physical constants of light linear hydrocarbones. $x^{*} \equiv$ solubility in water from $[7] ; T_{b} \equiv$ boiling temperature; $D_{m} \equiv$ mass diffusion coefficient in water from [8].

\begin{tabular}{|c|c|c|c|}
\hline & $\begin{array}{c}x^{*} \\
(\mathrm{~mol} / \mathrm{mol})\end{array}$ & $\begin{array}{c}T_{b} \\
\left({ }^{\circ} \mathrm{C}\right)\end{array}$ & $\begin{array}{c}D_{m} \\
\left(10^{-9} \mathrm{~m}^{2} / \mathrm{s}\right)\end{array}$ \\
\hline C5 & $1.0 \cdot 10^{-5}$ & 35 & $1.06 \pm 0.12$ \\
C6 & $2.1 \cdot 10^{-6}$ & 69 & $1.05 \pm 0.41$ \\
C7 & $5.4 \cdot 10^{-7}$ & 99 & - \\
\hline
\end{tabular}

than the atmospheric pressure: three alkanes have been used for this study: pentane, hexane and heptane. The solubility of those alkanes $x^{*}$ in water is very low [7]; values for the solubility found in the literature are given in Table 1.

Specific interaction: A specific interaction of the alkane molecules with the clay platelets, leading for instance to the adsorption of the alkane on the surface of the platelets, may increase the apparent solubility of the oil [9]. However, using Chemical Oxygen Demand test, we did not measure any significant change in the solubility of alkane in a Laponite dispersion compared to the one in water. Due to the large surface-to-volume ratio of the clay platelets (it is of the order of $\simeq 10^{8} \mathrm{~m}^{2} / \mathrm{m}^{3}$ for a mass fraction $\phi_{m} \simeq 1 \%$ ), any adsorption of the alkane on the surface of the platelets would lead to a large concentration in alkane even after the evaporation of the oil from the emulsion. For instance, assuming close packing adsorption of alkane, one would find $c_{\text {ads }} \simeq 10^{-3} \mathrm{~mol} / \mathrm{g}$ of dispersion which is significantly larger than the solubility of the alkane in the aqueous phase (from Tab. 1, the solubility of pentane is $c^{*}=6 \cdot 10^{-7} \mathrm{~mol} / \mathrm{g}$ of water). In the following, we assume that the solubility of alkane in a Laponite dispersion is the same as in water.

Emulsification process: Emulsions are prepared using an ultrasonic emulsifier without adding any surfactant and they are stable for several weeks. During the emulsification process, the Laponite dispersion is rejuvenated by the strong shear applied but becomes pasty again when at rest. Emulsions are polydisperse, the typical size of the emulsified droplets is $a_{0} \simeq 20 \mu \mathrm{m}$ and variance $\sigma_{a} \simeq 10 \mu \mathrm{m}$. The volume fraction of the emulsion is varied between $1 \%$ and $10 \%$. It is determined a priori by weighting the different compound and it has been checked $a$ posteriori: a known volume of the sample has been scanned with an optical microscope, and the number and size of the droplets have been measured. Error on the volume fraction is larger for the more volatile compound but it is less than $10 \%$ for pentane whereas it is less than $1 \%$ for heptane.

\section{Stabilization mechanisms}

Qualitative observations: The stability of the emulsions has been tested by varying the mass fraction of Laponite in the emulsifying phase while the volume fraction of the oil phase has been kept constant at $\phi_{v}=1 \%$. We observe that for concentration in Laponite smaller than $\phi_{m} \simeq 2 \% \mathrm{w} / \mathrm{w}$, the oil droplets cream and fur- ther separate into an oil phase; on the contrary, when the Laponite is concentrated enough, the oil droplets remain well dispersed within the aqueous phase over large time scales (a few months). The critical concentration in Laponite corresponds to the one for which Laponite dispersions exhibit a yield stress that is large enough to counterbalance buoyancy stresses $[5,10,11]$. The yield stress needed to prevent a $10 \mu \mathrm{m}$ droplet from creaming is $F_{b} / S=\frac{4}{3} \Delta \rho g R \simeq 0.1 \mathrm{~Pa}$. Emulsions are mechanically stable when the yield stress $\sigma_{Y}$ is comparable to this stress $F_{b} / S$ [12]. Oil droplets therefore remain well dispersed in the Laponite provided the yield stress reaches such a value within time scales short compared to the characteristic time of creaming. The stress values and time scales at stake are consistent with values found in the literature $[13,14]$.

A question that naturally arises concerning the stability of the emulsions is whether the Laponite platelets play a role in it. Some emulsions - called Pickering emulsions - are known to be stabilized by the trapping of nanoparticules at the oil/water interfaces. Moreover, Pickering adsorption has been invoked to explain the stability of some emulsions in Laponite dispersions [15-18]. However, if Pickering stabilization was significant in our case, it should prevent the droplets from coalescence for much smaller concentration in Laponite. Note that in any case the Laponite platelets are in large excess, the concentration needed for the total coverage of the oil/water interfaces being $\phi_{m} \sim 0.001 \%$, which is far smaller than the critical mass fraction we have found. Indeed, we clearly observe that the emulsions are stable only in the presence of a yield stress.

\section{Evaporation process}

Evaporation of the emulsions takes place in open air, which means that the atmosphere in contact with the emulsion is never saturated in alkane (we will see a justification of that later). It is well known that the transport of the volatile alkane in the atmosphere takes place by a diffusive process through an hydrodynamical boundary layer which is of the order of $1 \mathrm{~mm}$ and then by advection due to air draft. Optical observation of the evaporation of the emulsions has been performed using microscopic technics. For the observation purpose, the emulsion is quenched between two glass slabs $100 \mu \mathrm{m}$ apart. Typical micrographs are shown in Figures 1(a), (b) and (c).

The striking feature that appears on these pictures is that a sharp front forms in the vicinity of the interface with the atmosphere. The front separates a droplet-free phase, which is in contact with the atmosphere, from the emulsion. As time elapses, this front moves away from the interface. Quantitative measurements of the front displacement have been performed in the $1 \mathrm{D}$ geometry of a capillary tube. Since we focus on the evaporation of the alkane phase only, a small volume of silicon oil is inserted between the emulsion and the atmosphere. Silicon oil is miscible with alkane in all proportion whereas it is not miscible with water. The silicon oil volume therefore acts 


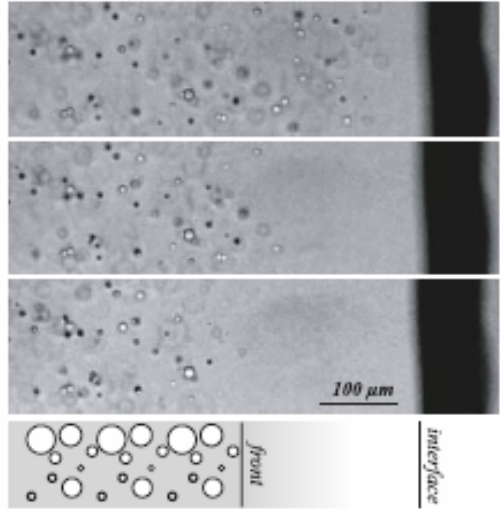

Fig. 1. Photographs of an emulsion of pentane at room temperature taken at time intervals of 45 minutes. The emulsion appears on the upper part of the photograph. A sharp front separating the emulsion from the droplet-free Laponite dispersion develops from the interface with the atmosphere (on the right of the picture)

as a cork that prevents evaporation for water but not for the alkane. Water evaporation would indeed modify the evaporation process of the emulsion as emphasized in previous works $[2,3]$. It has been checked experimentaly that the addition of the silioon oil does not change the kinetics of displacement of the front at a short time scale when evaporation of water is negligible. Therefore, evaporation of the alkane is the same in the vicinity of an interface emulsion/silioon oil as of an interface emulsion/air. In the following, the "interface" therefore refers to the emulsion/silicon oil, that has the same properties regarding the transport of alkane as an interface emulsion/air. As in the example shown in Figure 2, the variation with time of the distance between the front and the interface, $L(t)$, follows a power law with an exponent $1 / 2$. We have observed a similar behavior whatever the nature of the alkane. The evaporation kinetics of the oil phase therefore presents a diffusive behavior in each case.

Thermodynamics: The alkane contained in the droplets of the emulsion is pressurized by the effect of capillarity and the equilibrium concentration in alkane at the interface of the droplets is shifted from equilibrium values in the bulk (this mechanism is known to be responsible for Ostwald ripening in emulsions [1]). An extra overpressure may also result from the stress exerted by the Laponite on the droplet but this effect will not be considered here. The atmosphere with which the emulsion is in contact is not saturated in alkane either at the beginning of, or during the evaporation process, as shown in the following. Therefore, the chemical potential of the alkane being larger within the droplets than in the atmosphere, is favorable for the oil phase to empty out into the atmosphere.

\section{Diffusion-controlled evaporation model}

We denote as $x$ the molar fraction in alkane dissolved in the aqueous phase. In the following, we will assume that

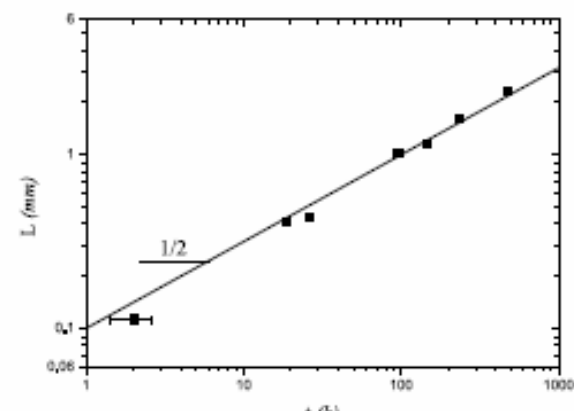

$t(k)$

Fig. 2. Distance between the front and the interface, $L$, as a function of time. The experimental data (squares) is well described by a diffusive law (full line) with an effective diffusion coefficient $D=3 \cdot 10^{-12} \mathrm{~m}^{2} / \mathrm{s}$. Emulsion of hexane at $\phi_{v}=3 \%$. The experiment lasts one month.

the transport is controlled by the difference between the concentration in the emulsion at the droplets interface $x^{D}$ and at the vapor interface $x^{V}$.

Equating the chemical potential of the alkane in the droplets to the chemical potential of the alkane dissolved in the aqueous phase, one finds the concentration at the corresponding interface $x^{D}$

$$
\frac{x^{D}}{x^{*}}=\exp \frac{a_{c}}{a},
$$

where $a$ is the radius of the droplet and $a_{c}=2 \gamma v_{m}^{C_{n}} / R T$, with $v_{m}^{C_{n}}$ the molar volume of the alkane. Necessarily: $x^{D}>x^{*}$. However, the difference in those values is small since, using values given in the literature ${ }^{2}$, we find $a_{c} \simeq$ $4 \mathrm{~nm}$ corresponding to a difference of the order of $0.1 \%$.

The concentration in alkane at the interface with the atmosphere is given by Henry's law:

$$
\frac{x^{V}}{x^{*}}=\frac{P_{v}}{P_{\text {sat }}} .
$$

Here, we always have $x^{V}<x^{*}$ and in practice, as we explained later, we can always assume $x^{V} \ll x^{*}$.

Let us now consider the equation of transport for the alkane within the Laponite dispersion. The diffusion of the alkane in the aqueous phase simply writes

$$
\frac{\partial x}{\partial t}=D_{m} \Delta x,
$$

where $D_{m}$ is the diffusion coefficient of the alkane in the laponite dispersion. Two boundary conditions have to be added to equation (3). First, at the interface between the droplets and the laponite, the concentration in alkane is $x=x^{D}$ given by equation (1). Second, at the interface, $x=x^{V}$ given by equation (2). Let us focus on equation (1). It means that as long as alkane droplets exist, the concentration in alkane in their vicinity is $x \simeq x^{D}$.

${ }^{2}$ From [19], the interfacial tension with water is $\gamma=48.41 \pm$ $0.44 \mathrm{mN} / \mathrm{m}$ for pentane and $\gamma=49.46 \pm 0.50 \mathrm{mN} / \mathrm{m}$ for hexane at $20^{\circ} \mathrm{C}$. 


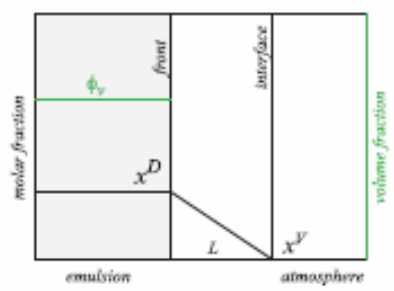

Fig. 3. Spatial variations of the droplet volume fraction and of the molar fraction of the dissolved alkane as considered in the description of the transport of alkane to the atmosphere.

In the emulsion, the average distance between the droplets is $d \equiv a_{0} / \sqrt[3]{\phi_{v}} \lesssim 100 \mu \mathrm{m}$ and, therefore, the characteristic time associated with diffusion is $\tau \sim 10 \mathrm{~s}$ (assuming a typical value of the mass diffusion coefficient $D_{m} \simeq 10^{-9} \mathrm{~m}^{2} / \mathrm{s}$ ). As this time is short compared to the time scale of the front displacement (of the order of $\tau \sim 10^{3} \mathrm{~s}$ ), the local concentration in alkane is the stationary one given by thermodynamics following equation (1). As schematized in Figure 3, there are thus two different length scales in the problem. The problem can therefore be described as follows: in the region where the concentration in droplets is large enough, we simply have $x=x^{D}$. In the absence of droplet, the concentration in alkane follows a one-dimensional diffusion equation similar to the equation $\partial_{t} x=D_{m} \partial_{x g} x$, with $x=x^{V}$ and $x=x^{D}$ at $z=0$ and $z=L$. Here, $z$ is the distance to the interface with the atmosphere.

It is crucial to note that the front between the emulsion and the droplet-free Laponite is very sharp and does not broaden with time. Its stability results from the fact that, owing to surface tension effects, the smaller a droplet, the faster the alkane it contains dissolves into the aqueous phase. Thus, when the front moves, the size of the droplets decreases, which leads to an increase of the capillary pressure inside them and acoelerates the leaking of the alkane away from the droplets. The concentration in droplets as a function of the distance to the interface can then be reasonably described by a step function. In that case, the displacement of the front is related to the flux through the conservation of mass

$$
j=\frac{\phi_{v}}{F} \frac{\mathrm{d} L}{\mathrm{~d} t},
$$

where $F \equiv\left(v_{m}^{C_{n}} / v_{m}^{\mathrm{H}_{2} \mathrm{O}}\right)$ is a numerical prefactor that ensures conversion from volume fraction to molar fraction.

Within our model, the oncentration profile can be assumed to be quasi-stationary with fixed values of the concentration at the front and at the interface. The flux towards the interface then reads (see Fig. 3)

$$
j=D_{m} \frac{\delta x}{L},
$$

with $\delta x=x^{D}-x^{V}$. As pointed out in what precedes, the droplets being rather large, $x^{D} \simeq x^{*}$. As the atmosphere is undersaturated in alkane, $x^{V} \simeq 0$. Therefore, equations (1) and (2) give $\delta x \simeq x^{*}$.
Integrating the first-order equation resulting from equations (4) and (5), our model predicts that the front displacement follows a diffusive power law $L=\sqrt{2 D t}$, with

$$
D=\frac{F x^{*}}{\phi_{v}} D_{m} .
$$

As expected, the effective diffusion coefficient $D$ is proportional to the solubility of the oil $x^{*}$. Note that because $F x^{*} / \phi_{v} \ll 1$, the effective diffusion coefficient $D$ is very small compared to $D_{m}$. Thus, the assumption of a quasi-stationary concentration profile is consistent with our description. The $D \propto \phi_{v}{ }^{-1}$ dependence is not intuitive, it can however be simply understood: during the evaporation process, the droplets play the role of a reservoir that feeds the flux of oil towards the interface. The larger the volume fraction $\phi_{v}$, the larger this reservoir and therefore, the slower the front moves away from the interface with the atmosphere. Furthermore, the increase in the front velocity with the oil volume fraction leads to the formation of a shock wave that is analogous to the one observed for instance in sedimentation of suspensions of monodisperse spherical solid particles. In the latter case, the settling velocity increases for decreasing particle concentration, which results in a sharp front separating the suspension from the particle-free fluid that is very similar to the one we observe during the evaporation of emulsions.

Finally, we can now consider the assumption made that $x^{V} \simeq 0$. The alkane flux through the emulsifying phase is $j_{\text {lap }}=\left(M_{C_{n}} / v_{m}^{\mathrm{H}_{2} \mathrm{O}}\right) \cdot\left(D_{m} \delta x\right) / L$. Let us compare that flux with the one in the atmosphere. Assuming diffusion-controlled transport yields: $j_{\text {atm }}=\left(M_{C_{n}} / v_{m}^{G}\right)$. $\left(D_{m}^{G} x^{V}\right) / \Lambda$, where $A$ is the typical length over which the concentration gradient establishes in the atmosphere. Using the classical electric analogy, both equations can be interpreted as electric resistances subject to a difference of potential (corresponding to $\delta x$ ), which induces a current analogous to the alkane flux. The resistances in the Laponite and in the atmosphere can therefore be defined such that: $R_{\text {lap }}=\left(L v_{m}^{\mathrm{H}_{2} \mathrm{O}} / M_{C_{n}} D_{m}\right)$ and $R_{\text {lap }}=$ $\left(A v_{m}^{G} / M_{C_{n}} D_{m}^{G}\right)$. The resistance in the Laponite is larger than the one in the atmosphere provided $A<L\left(D_{m}^{G} / D_{m}\right)$ $\left(v_{m}^{\mathrm{H}_{2} \mathrm{O}} / v_{m}^{G}\right)$, which leads to $A<3 L$ (the mass diffusion coefficient of alkanes in the gas phase is [20]: for pentane, $D_{m}^{G}=3.7 \cdot 10^{-6} \mathrm{~m}^{2} / \mathrm{s}$ ). In our experimental conditions where the atmosphere is the one of an ordinary room, $A$ is close to $1 \mathrm{~mm}$. $R_{\text {lap }}$ is therefore larger than $R_{\text {atm }}$ for almost all our experimental data. The system being analogous to the two resistances connected in series, the evaporation kinetics is therefore controlled by the diffusion of the dissolved oil through the aqueous phase. That finding is similar to the evaporation mechanism proposed in creamed emulsions [3].

\section{Experimental results}

If we refer to equation (6), the effective diffusion coefficient of the front $D$ should only depend on the solubility of the emulsified oil $x^{*}$ and on the volume fraction of the 


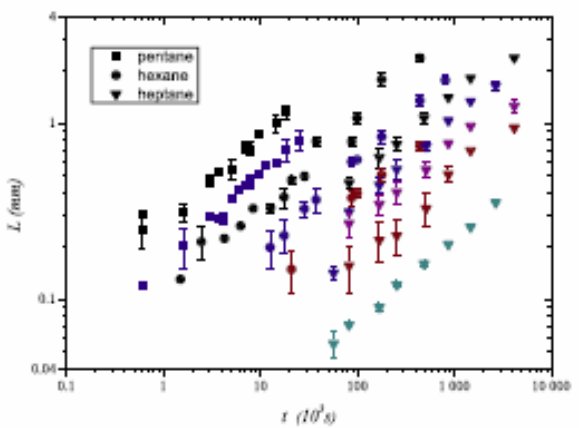

(a) Raw measurements

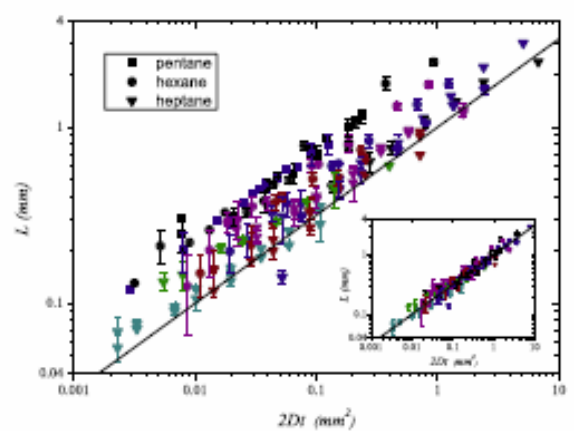

(b) Rescaled lengths

Fig. 4. (Color online) (a) Distance of the front from the interface as a function of time. The time variations of $L$ are shown for three different alkanes (different symbols) and when varying the volume fraction in oil of the emulsions $\phi_{v}$ (different colors). (b) Rescaled lengths assuming $D_{m}=1 \cdot 10^{-9} \mathrm{~m}^{2} / \mathrm{s}$ (insert: the collapse of data is better by assuming for $D_{m}$ the values given in Tab. 2). The continuous line represents $\sqrt{2 D t}$.

Table 2. Mass diffusion coefficient $D_{m}\left(10^{-9} \mathrm{~m}^{2} / \mathrm{s}\right)$ (a): from [8]; (b): present study.

\begin{tabular}{|c|c|c|}
\hline & $D_{m}{ }^{(a)}$ & $D_{m}{ }^{(b)}$ \\
\hline C5 & $1.06 \pm 0.12$ & $6 \pm 1$ \\
C6 & $1.05 \pm 0.41$ & $2.0 \pm 0.5$ \\
C7 & - & $1.3 \pm 0.25$ \\
\hline
\end{tabular}

emulsion $\phi_{v}$. In Figure 4(a), we report measurements when those two parameters are varied. On Figure 4(b), we see that the same data collapse onto on a single curve when $L$ is plotted as a function of $2 D t$, with $D$ given by equation (6). $D$ in particular depends on the mass diffusion coefficient of the alkane, $D_{m}$, which is a poorly known quantity. We have used the value of the mass diffusion coefficient of light alkanes in water currently found in the literature [8]: $D_{m} \simeq 10^{-9} \mathrm{~m}^{2} / \mathrm{s}$.

The collapse of the data onto a master curve in the representation of Figure 4(b) is remarkable given the large spread of the initial data of Figure 4(a). It validates in particular our description of the evaporation process, limited by the diffusion of the dissolved alkane through the emulsifying phase. The somewhat larger values found experimentally as compared to $\sqrt{2 D t}$, especially in the cases of pentane and hexane, doubtlessly result from the approximate value of the mass diffusion coefficient we have used Owing to its weak concentration (particle volume fraction close to $1 \%$ ), the presence of Laponite in the emulsifying phase is not likely to induce a significant variation of the mass diffusion coefficient as compared to the one in water, however $D_{m}$ should depend on the nature of the alkane.

This point clearly appears on Figure 5 where the effective diffusion coefficient has been normalized by a quantity taking into account the alkane solubility and molar volume and is shown as a function of $\phi_{v}$. The $D \propto \phi_{v}{ }^{-1}$ dependency is well observed. The differences for the three

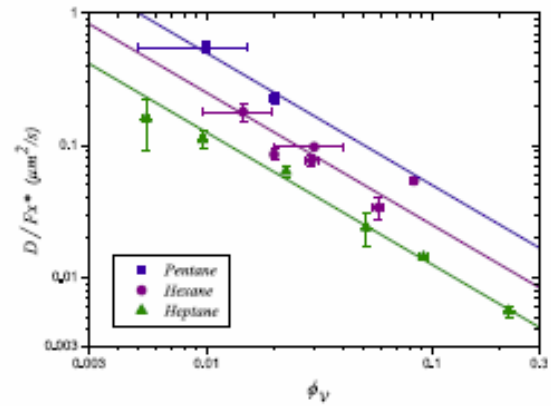

Fig. 5. Effect of the volume fraction of the emulsion $\phi_{v}$. As the effective diffusion coefficient spreads over several orders of magnitude depending on the alkane, it has been rescaled by the solubility and by the molar volume of each alkane $D \rightarrow D / F x^{*}$, where $F=v_{m}^{C n} / v_{m}^{H_{2} \mathrm{O}}$. Straight lines stand for $D=\vec{D}_{m} / \phi_{v}$.

alkanes can only be attributed to different values of $D_{m}$, that can thus be extrapolated. Table 2 compares the values of the mass coefficient $D_{m}$ of alkanes in a Laponite dispersion to data available in the literature $[8,21]$ for diffusion of alkanes in water. Indeed, in our experiment, the evaluation of the mass diffusion coefficient is not direct as in a NMR measurement. However the large differences observed are intriguing and more experimental data in both water and Laponite is needed to draw conclusions.

\section{Conclusion}

We present herein a work dealing with emulsions that are mechanically stabilized, the emulsifying phase presenting a yield stress that prevents the oil droplets from creaming. We have studied the evaporation of the oil phase from such an emulsion. The displacement of the front formed during 
the evaporation process has been measured as a function of time for a large set of parameters. We show that the experimental data is well described assuming that the oil transport is controlled by the diffusion of the dissolved oil through the aqueous phase. The variation of the effective diffusion coefficient of the oil with the oil volume fraction in the emulsion is in particular well captured by the model we develop. We also propose a mechanism explaining the formation of a sharp front between the emulsion and the droplet-free aqueous phase. These results shed new light on the evaporation process of emulsions, which have been poorly studied in the past.

\section{References}

1. A.S. Kabalnov, A.V. Pertzov, E.D. Shchukin, J. Colloid Interface Sci. 118, 590 (1987).

2. I. Aranberri, B.P. Binks, J.H. Clint, P.D.I. Fletcher, Langmuir 20, 2069 (2004).

3. I. Aranberri, K. Beverley, B. Binks, J. Clint, P. Fletcher, Langmuir 18, 3471 (2002).

4. S. Cocard, J.-F. Tassin, T. Nicolai, J. Rheol. 44, 585 (2000).

5. P. Mongondry, J.-F. Tassin, T. Nicolai, J. Colloid Interface Sci. 283, 397 (2005).

6. D. Bonn, S. Tanase, B. Abou, H. Tanaka, J. Jacques Meunier, Phys. Rev. Lett. 89, 015701 (2002).
7. C. Mc Auliffe, Sciences 163, 478 (1969).

8. W. Price, O. Söderman, J. Phys. Chem. A 104, 5892 (2000).

9. A.L. Cheng, W.L. Huang, Org. Geochem. 35, 413 (2004).

10. A. Mourchid, A. Delville, J. Lambard, E. Lecolier, P. Levitz, Langmuir 11, 1942 (1995).

11. P. Levitz, E. Lecolier, A. Mourchid, A. Delville, S. Lyonnard, Europhys. Lett. 49, 672 (2000).

12. N. Dubash, I.A. Frigaard, J. Non-Newtonian Fluid Mech. 142, 123 (2007).

13. C. Wilhelm, J. Elias, F. Browaeys, A. Ponton, J.-C. Bacri, Phys. Rev. E 66, 021502 (2002).

14. B. Gueslin, L. Talini, B. Herzhaft, Y. Peysson, C. Allain, Phys. Fluids 18, 103101 (2006).

15. J. Thieme, S. Abend, G. Lagaly, Colloid Polym. Sci. 277, 257 (1999).

16. N.P. Ashby, B.P. Binks, Phys. Chem. Chem. Phys. 2, 5640 (2000).

17. L. Torres, R. Iturbe, M. Snowden, B. Chowdhry, S. Leharne, Colloid Surf. A 302, 439 (2007).

18. F. Yang, Q. Niu, D. Sun, J. Colloid Interface Sci. 306, 285 (2007).

19. Y. Morl, N. Tsul, M. Klyomlyat, J. Chem. Eng. Data 29, 407 (1984)

20. S. Chapman, T.G. Cowling, The Mathematical Theory of Non Uniform Gases, third edition (Cambridge University Press, 1970).

21. A. Laaksonen, P. Stilbs, Mol. Phys. 74, 747 (1991). 